\title{
Estimación de Radiación Solar Horaria Utilizando Modelos Empíricos y Redes Neuronales Artificiales
}

Víctor Adrián Jiménez, ${ }^{\text {A Adrián Willi \& Sebastián Rodríguez }}{ }^{\mathrm{iii}}$

\section{Resumen}

La radiación solar es uno de los parámetros más importantes para el desarrollo de aplicaciones e investigaciones relacionadas a energías renovables. Sin embargo, la adquisición de mediciones de radiación solar no siempre es posible por diferentes motivos y es necesario contar con modelos que permitan estimarla. Estos modelos en su mayoría utilizan variables climáticas difíciles de medir y que no siempre están disponibles en todos los sitios. El objetivo de este trabajo es aplicar un método para estimar radiación solar horaria, basado en redes neuronales, utilizando como variables de entrada estimaciones de radiación solar obtenidas mediante un modelo matemático simple y variables climáticas de fácil adquisición: Temperatura, Presión y Humedad. Además, para comprobar que las redes neuronales son más adecuadas en estos casos se hizo una comparativa con estimaciones realizadas con regresión lineal. Los modelos generados fueron ajustados y validados con datos provenientes de cinco estaciones meteorológicas de la provincia de Tucumán, Argentina, logrando obtener en promedio un error de 11.0\% (Root Mean Squared Error) con regresión lineal y $7.84 \%$ con redes neuronales.

Palabras claves: radiación solar horaria, modelos empíricos, redes neuronales feedforward, regresión lineal, energía renovable.

Universidad Tecnológica Nacional - Facultad Regional Tucumán - Grupo de Investigación en Tecnologías Informáticas Avanzadas. Argentina

ii Universidad Tecnológica Nacional - Facultad Regional Tucumán - Grupo de Investigación en Tecnologías Informáticas Avanzadas. Argentina

iii Universidad Tecnológica Nacional - Facultad Regional Tucumán - Grupo de Investigación en Tecnologías Informáticas Avanzadas. Argentina 


\begin{abstract}
Solar radiation is one of the most important parameter for application, development and research related to renewable energy. However, the solar radiation measurements acquisition is not always possible for many reasons, and it is necessary to have models to estimate it. Mostly, these models use climatic variables that are difficult to measure and are not always available in all sites. The aim of this paper is to estimate hourly solar radiation, based on neural networks, using solar radiation estimates obtained by a simple mathematical model and climatic variables easy to acquire as input variables: Temperature, pressure and humidity. In addition, to verify that neural networks are most appropriate in these cases, a comparison with linear regression estimates was made. The models were adjusted and validated with data from five weather stations of the province of Tucuman, Argentina, achieving on average a Root Mean Squared Error value of $11.0 \%$ for linear regression and $7.84 \%$ for neural networks.
\end{abstract}

Keywords: hourly solar radiation, empirical models, feedforward neural networks, linear regression, renewable energy.

Fecha de recepción 27/09/2016 - Fecha de aceptación 04/08/2017 


\section{Introducción}

La energía solar es la principal, la más grande y la más importante fuente de energía renovable. Contar con datos de radiación solar es importante para el diseño, desarrollo y optimización de aplicaciones, como los sistemas con generadores fotovoltaicos (Gilani, Dimas, Shiraz, \& others, 2011), agrometeorología (Will, Bustos, Bocco, Gotay, \& Lamelas, 2013) y estimación de variables antropogénicas como el consumo eléctrico. En el caso de consumo eléctrico, la radiación solar es una variable muy importante ya que en países donde la temperatura ambiente es alta y la irradiación solar en verano es fuerte, la temperatura del devanado de los transformadores de distribución podría ser superior a la máxima admisible, lo que reduce la vida útil del mismo (Gorgan et al., 2012) . Sin embargo, las mediciones de radiación solar en un área específica no siempre se encuentran disponibles debido al alto costo y los requerimientos tecnológicos (Teke, Yıldırım, \& Çelik, 2015). La falta de datos en bases de datos climáticas es un problema sumamente común en todo el mundo. Además, las fallas en la toma de datos de las estaciones meteorológicas disponibles siguen sucediendo, ya que por lo general se encuentran en lugares remotos y el acceso para su reparación y/o mantenimiento muchas veces resulta difícil. Ya sea que no se cuenta con una estación meteorológica cercana, o que los datos disponibles presenten muchos valores faltantes o anómalos, es necesario contar con un método que permita estimar la radiación solar.

En la literatura podemos encontrar una amplia variedad de métodos para estimar radiación solar a nivel horario. Existen modelos empíricos (Gilani et al., 2011; Noorian, Moradi, \& Kamali, 2008; Pandey \& Soupir, 2012; Spokas \& Forcella,Frank, 2006), métodos estadísticos (Ji, Chan, Loh, \& Chen, 2009; Reikard, 2009), basados en regresión lineal y no lineal (Elminir, Azzam, \& Younes, 2007; Furlan, De Oliveira, Soares, Codato, \& Escobedo, 2012) y métodos basados en técnicas de inteligencia artificial (Sfetsos \& Coonick, 2000), donde las Redes Neuronales Artificiales son las más utilizadas (Cao \& Lin, 2008; HernándezTravieso, Travieso, Alonso, \& Dutta, 2014; Khatib, Mohamed, Sopian, \& Mahmoud, 2012; Mellit, Eleuch, Benghanem, Elaoun, \& Pavan, 2010; Sayago, Bocco, Ovando, $\&$ Willington, 2011). Muchos de estos métodos modelan la radiación solar a partir de diferentes variables y parámetros disponibles, desde los más simples que utilizan parámetros geográficos (latitud, longitud, altitud, etc.) hasta los más complejos que requieren una o varias variables meteorológicas (temperatura ambiente, nubosidad, humedad, presión atmosférica, heliofanía, etc.). Una completa revisión de estos y otros métodos de estimación de radiación solar puede encontrarse en (Ahmad \& Tiwari, 2011; Teke et al., 2015; Yadav \& Chandel, 2014).

En un trabajo previo (Jimenez, Barrionuevo, Will, \& Rodriguez, 2016) se propuso una metodología para estimar radiación solar global diaria basada en el uso de un modelo empírico en combinación con redes neuronales artificiales, logrando buenos resultados 
con bajos niveles de error. El método utiliza parámetros geográficos y variables climáticas de fácil acceso en cualquier región. Se propone como objetivo para este trabajo continuar con la misma línea de investigación y determinar si dicha metodología es adecuada para estimar radiación solar horaria. Para ello se utiliza un modelo matemático simple para generar estimaciones iniciales de radiación solar a nivel horario, que luego servirán como entradas a una red neuronal junto a otras variables climáticas: temperatura, humedad y presión atmosférica. El método se aplicó y validó con datos de cinco bases meteorológicas localizadas en la provincia de Tucumán, Argentina.

El resto del trabajo se organiza de la siguiente manera: la Sección 2 describe los datos utilizados y detalla la metodología empleada; En las Secciones 3 y 4 se describen las pruebas realizadas y los resultados obtenidos respectivamente; Por último, en la Sección 5 se presentan las conclusiones del trabajo.

\section{Materiales y Métodos}

\subsection{Datos utilizados}

Los datos climáticos utilizados en este trabajo fueron recolectados de cinco bases meteorológicas, pertenecientes a la Estación Experimental Agroindustrial Obispo Colombres (E.E.A.O.C.), distribuidas en la provincia de Tucumán, Argentina (las coordenadas se muestran en la Tabla 1). Los datos utilizados corresponden a valores medios de mediciones tomadas en intervalos de 15 minutos, en el período comprendido entre 01/01/2012 y 20/11/2013. Entre todas las variables provistas por las estaciones meteorológicas, en este trabajo utilizamos sólo aquellas que son fácilmente medibles en sitios donde no se cuenta con bases meteorológicas cercanas. Estas variables son:

- Temperatura externa $\left[{ }^{\circ} \mathrm{C}\right]$

- Humedad relativa [\%]

- Presión atmosférica $[\mathrm{hPa}]$

- Radiación solar observada [W/m2]

Tabla 1: Descripción de las Estaciones Meteorológicas utilizadas.

\begin{tabular}{|c|c|c|}
\hline Estación Meteorológica & Posición Geográfica & \begin{tabular}{|l|} 
Registros \\
Dañados [\%]
\end{tabular} \\
\hline Santa Ana & Lat. $27^{\circ} 47^{\prime} 21^{\prime \prime} \mathrm{S}$ - Lon.65 $40^{\prime} 30^{\prime \prime} \mathrm{O}$ & 2.52 \\
\hline Pueblo Viejo & Lat. $27^{\circ} 11^{\prime} 56^{\prime \prime} \mathrm{S}$ - Lon. $27^{\circ} 11^{\prime} 56^{\prime \prime} \mathrm{O}$ & 10.84 \\
\hline Monte Redondo & Lat. $65^{\circ} 37^{\prime} 10^{\prime \prime} \mathrm{S}$ - Lon.64 50' 58" O & 0.07 \\
\hline El Colmenar & Lat. $26^{\circ} 47^{\prime} 21^{\prime \prime} \mathrm{S}$ - Lon. $65^{\circ} 11^{\prime} 13^{\prime \prime} \mathrm{O}$ & 0.08 \\
\hline Casas Viejas & Lat. $27^{\circ} 46^{\prime} 56^{\prime \prime} \mathrm{S}$ - Lon.65 $30^{\prime} 24^{\prime \prime} \mathrm{O}$ & 0.06 \\
\hline
\end{tabular}


En un análisis inicial de los datos, y como es usual en mediciones tomadas con redes de sensores distribuidas, se encontraron registros con valores faltantes o erróneos (fuera de rango), que abarcan desde pocas horas a algunos días. Esto se debe posiblemente a problemas en los equipos de medición o en los procesos de adquisición de información. Sin embargo, debido a que la cantidad de datos afectados no es significativa, se optó por eliminar los registros completos que presenten alguna anomalía. El porcentaje de datos descartados en cada base meteorológica se detalla en la Tabla 1.

\subsection{Modelo Empírico}

Gran parte de los métodos empíricos encontrados en la literatura utilizan relaciones empíricas para estimar la radiación solar global a partir de parámetros del lugar y algunas variables climáticas. Sin embargo, debido a su simplicidad en este trabajo utilizamos el procedimiento basado en el método propuesto por Spokas y Forcella (Gilani et al., 2011; Spokas \& Forcella,Frank, 2006). Este propone un modelo para la energía de radiación total que incide en la superficie de la tierra $\mathrm{G}_{h}$, la cual puede descomponerse en dos partes: radiación de haz directo $\mathrm{G}_{B h}$ y la radiación solar difusa $\mathrm{G}_{D h}$. La radiación solar total es entonces la suma de estas dos componentes:

$$
\mathrm{G}_{\mathrm{h}}=\mathrm{G}_{\mathrm{Bh}}+\mathrm{G}_{\mathrm{Dh}}
$$

La intensidad de la radiación de haz directo $\mathrm{G}_{B h}$ es determinada por el ángulo que forma la dirección del sol y la normal a la superficie (llamado ángulo cenital $\theta_{z}$ ) y la intensidad de radiación recibida en forma perpendicular a la superficie $\mathrm{G}_{P h}$, de acuerdo a la Ecuación 2 . El ángulo cenital varía temporal y geográficamente, pero puede ser calculado en función de la hora angular $\omega=15 \cdot(h r-12.5)\left[{ }^{\circ} \mathrm{C}\right]$, la latitud $\varphi$ y el día del año doy según las Ecuaciones 3 y 4 . La intensidad $\mathrm{G}_{P h}$ a su vez puede ser calculada a partir de un modelo empírico dado por la Ecuación 5, donde $K=$ $1.360[\mathrm{KW} / \mathrm{m} 2]$ es la constante solar, $\tau$ es la transmitancia atmosférica y $\mathrm{P}_{a}$ es la presión atmosférica $[\mathrm{hPa}]$. La transmitancia atmosférica puede calcularse a partir de datos de humedad relativa $\mathrm{H}_{R}$ según la Ecuación 7.

$$
\begin{gathered}
\mathrm{G}_{\mathrm{Bh}}=\mathrm{G}_{\mathrm{Ph}} \cos \left(\theta_{\mathrm{z}}\right) \\
\cos \left(\theta_{\mathrm{z}}\right)=\sin (\varphi) \sin (\delta)+\cos (\varphi) \cos (\delta) \cos (\omega) \\
\delta=23,45 \cdot \sin (6,303(284+\text { doy }) / 365)\left[{ }^{\circ} \mathrm{C}\right] \\
\mathrm{G}_{\mathrm{Ph}}=\mathrm{K} \cdot \tau^{\mathrm{m}} ; \mathrm{m}=\mathrm{P}_{\mathrm{a}} / 101,3 \cdot \cos \left(\theta_{\mathrm{z}}\right)
\end{gathered}
$$


La radiación solar difusa $\mathrm{G}_{\mathrm{Dh}}$, por otro lado, es aquella que es dispersada en la atmósfera después de ser absorbida por gases atmosféricos, nubes y partículas de polvo. La misma puede calcularse a partir del modelo descrito por la Ecuación 6 .

$$
\begin{gathered}
G_{D h}=0,30 \cdot K\left(1-\tau^{m}\right) \cos \left(\theta_{z}\right) \\
\tau=0,5\left[1-\left(1+\exp \left(64-H_{-} R\right) / 10\right)\right]+0,2
\end{gathered}
$$

Entonces, las variables y parámetros de entrada que requiere este modelo matemático son: hora del día $(h r)$, día del año $(d o y)$, presión atmosférica $\left(P_{a}\right)$, humedad relativa $\left(H_{R}\right)$ y latitud del sitio $(\varphi)$.

\subsection{Redes Neuronales Feedforward-Backpropagation}

Las redes tipo Feedforward Multicapa son uno de los métodos más conocidos y ampliamente utilizados para la estimación de radiación solar (Hernández-Travieso et al., 2014; Khatib et al., 2012; Mellit et al., 2010; Sayago et al., 2011). Como se muestra en la Figura 1, las redes Feedforward constan de una serie de capas: la primera tiene una conexión directa con los datos de entrada, y pueden ser conectadas a una o más capas ocultas o directamente a la capa de salida. En este tipo de redes las neuronas de una capa se conectan con todas las neuronas de la capa siguiente, y no existe ninguna retroalimentación. Estas redes son populares debido la flexibilidad de su estructura y porque son aproximadores universales: Una red Feedforward de una sola capa oculta con un número suficiente de neuronas es capaz de aproximar cualquier función continua con el nivel de precisión deseada (Sharma \& Chandra, 2010).

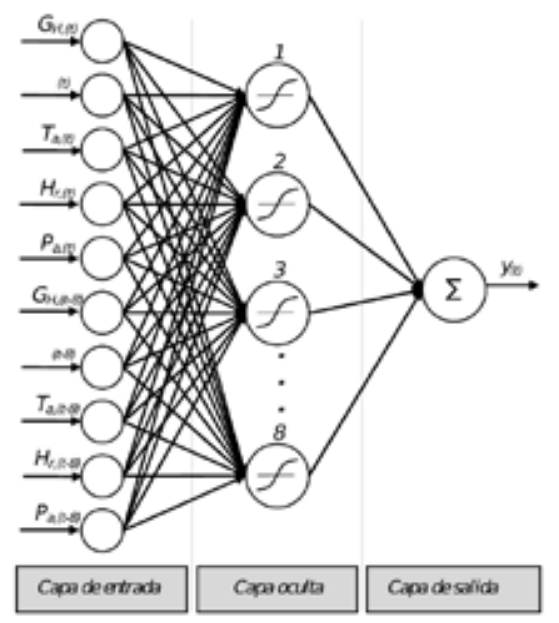

Figura 1: Esquema de la arquitectura de una red Feedforward de una capa oculta utilizada para estimar radiación solar. 
Para el desarrollo de este trabajo se optó por el uso de este tipo de red con 8 neuronas y función de transferencia sigmoidal Tangente Hiperbólica en la capa oculta, y función de transferencia Lineal para la neurona de la capa de salida. Para entrenar la red neuronal se utilizó el algoritmo Levenberg-Marquardt Backpropagation (Yu \& Wilamowski, 2011), debido a su gran eficiencia y rápida convergencia, aunque sus requisitos computacionales sean elevados (Hagan \& Menhaj, 1994). Se utilizaron datos del 2012 en el proceso de entrenamiento, dejando los datos del 2013 para validación del método. Se impuso 50 iteraciones como máximo para la condición de parada del entrenamiento.

El vector de entrada de la red se compone de la estimación inicial de la radiación solar global $\left(G_{h}\right)$ calculada con el modelo empírico descrito en la Sección 2.2, el ángulo cenital $\left(\theta_{z}\right)$ expresado en radianes calculado con la Ecuación 3, y variables climáticas: Temperatura $T_{a}$, Humedad relativa $H_{r}$ y Presión atmosférica $P_{a}$. Adicionalmente, para mejorar la precisión de las estimaciones realizadas con la red neuronal se incluyen variables correspondientes a valores en instantes anteriores (llamadas lagged variables en la literatura). Según pruebas preliminares se determinó que se aporta una mejora significativa agregando sólo variables de mediciones actuales (instante $t$ ) y las correspondientes a dos horas atrás (para el instante $t-8$ ). De este modo, la cantidad de variables de entrada utilizadas asciende a 10 en total (ver Figura 1).

Por otro lado, debido a que los valores de las variables utilizadas poseen diferentes magnitudes se decide normalizar los datos aplicando un escalado minmax a $[-1,1]$. De esta forma evitamos que el algoritmo de entrenamiento tenga preferencia por alguna de las variables utilizadas.

Tabla 2: Niveles de error alcanzados en cada conjunto de datos.

\begin{tabular}{|c|c|c|c|c|c|c|c|c|c|}
\hline \multirow{2}{*}{$\begin{array}{l}\text { Método de } \\
\text { Estimación }\end{array}$} & \multirow{2}{*}{$\begin{array}{l}\text { Estación } \\
\text { Meteoroló- } \\
\text { gica }\end{array}$} & \multicolumn{4}{|c|}{ Entrenamiento - Datos del 2012} & \multicolumn{4}{|c|}{ Validación - Datos del 2013} \\
\hline & & RMSE & $\mathrm{R}$ & MBE & RMSE\% & RMSE & $\mathrm{R}$ & MBE & RMSE\% \\
\hline \multirow{5}{*}{$\begin{array}{l}\text { Modelo } \\
\text { Empírico }\end{array}$} & $\begin{array}{l}\text { Casas } \\
\text { Viejas }\end{array}$ & 392.53 & 0.775 & -308.6 & 29.40 & 360.01 & 0.786 & -280.5 & 28.10 \\
\hline & $\begin{array}{l}\text { El } \\
\text { Colmenar }\end{array}$ & 410.87 & 0.718 & -314.4 & 32.00 & 381.14 & 0.752 & -294.4 & 28.68 \\
\hline & $\begin{array}{l}\text { Monte } \\
\text { Redondo }\end{array}$ & 411.49 & 0.723 & -319.6 & 30.59 & 378.99 & 0.759 & -295.7 & 27.99 \\
\hline & $\begin{array}{l}\text { Pueblo } \\
\text { Viejo }\end{array}$ & 448.43 & 0.693 & -356.2 & 34.55 & 407.55 & 0.732 & -323.6 & 31.89 \\
\hline & Santa Ana & 430.35 & 0.746 & -349.9 & 34.07 & 400.93 & 0.769 & -327.1 & 29.55 \\
\hline
\end{tabular}

Continúa... 


\begin{tabular}{|c|c|c|c|c|c|c|c|c|c|}
\hline \multirow{6}{*}{$\begin{array}{l}\text { Modelo } \\
\text { Empírico y } \\
\text { Regresión } \\
\text { Lineal }\end{array}$} & $\begin{array}{l}\text { Casas } \\
\text { Viejas }\end{array}$ & 149.21 & 0.881 & -11.6 & 11.62 & 141.93 & 0.895 & -27.9 & 10.68 \\
\hline & $\begin{array}{l}\text { El } \\
\text { Colmenar }\end{array}$ & 152.42 & 0.878 & -15.2 & 11.42 & 141.85 & 0.899 & -18.3 & 11.07 \\
\hline & $\begin{array}{l}\text { Monte } \\
\text { Redondo }\end{array}$ & 157.58 & 0.867 & -16.1 & 11.72 & 151.06 & 0.880 & -21.6 & 11.16 \\
\hline & Pueblo & & & & & & & & \\
\hline & Viejo & 155.63 & 0.866 & -14.9 & 11.99 & 146.04 & 0.882 & -21.3 & 11.43 \\
\hline & Santa Ana & 158.16 & 0.868 & -19.3 & 12.52 & 147.86 & 0.878 & -27.5 & 10.90 \\
\hline \multirow{5}{*}{$\begin{array}{l}\text { Modelo } \\
\text { Empírico } \\
\text { y Red } \\
\text { Neuronal }\end{array}$} & $\begin{array}{l}\text { Casas } \\
\text { Viejas }\end{array}$ & 105.33 & 0.938 & 0.6 & 7.89 & 103.82 & 0.940 & 3.4 & 8.11 \\
\hline & $\begin{array}{l}\text { El Colme- } \\
\text { nar }\end{array}$ & 110.92 & 0.931 & 1.1 & 8.64 & 102.93 & 0.940 & -11.4 & 7.75 \\
\hline & $\begin{array}{l}\text { Monte } \\
\text { Redondo }\end{array}$ & 110.01 & 0.930 & 0.2 & 8.18 & 112.71 & 0.926 & -0.3 & 8.32 \\
\hline & $\begin{array}{l}\text { Pueblo } \\
\text { Viejo }\end{array}$ & 108.79 & 0.930 & 0.1 & 8.38 & 96.51 & 0.944 & -3.5 & 7.55 \\
\hline & Santa Ana & 107.09 & 0.934 & 0.3 & 8.48 & 101.06 & 0.939 & -6.5 & 7.45 \\
\hline
\end{tabular}

\section{Diseño Experimental}

El procedimiento seguido para realizar las estimaciones de radiación solar en un determinado sitio se detalla en la Figura 2. En primer lugar se utilizar un modelo empírico para obtener una estimación inicial de la radiación solar. Dependiendo del modelo seleccionado se deberán utilizar variables temporales (hora del día y día del año), parámetros que dependen del sitio (por ejemplo longitud, latitud, uso horario) y algunas variables climáticas. La radiación solar estimada de esta forma pasa a formar parte del vector de entrada de la red neuronal, junto a mediciones de otras variables climáticas tomadas localmente que pueden o no haber sido usadas por el modelo empírico, dando como resultado las estimaciones finales de radiación solar.

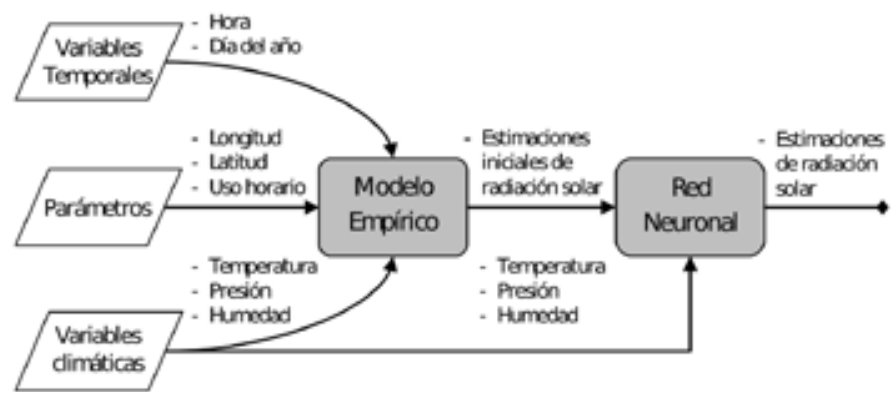

Figura 2: Procedimiento para estimar radiación solar combinando un modelo empírico y redes neuronales. 
En trabajos anteriores (Jimenez et al., 2016; Jimenez, Will, Rodriguez, \& Lamelas, 2014; Will et al., 2013) se comprobó que la radiación solar observada diaria presenta una relación lineal con otras variables climáticas, principalmente con temperatura, humedad, heliofanía y nubosidad, entre otras. Sin embargo, al no contar con todas estas variables usualmente utilizadas en la bibliografía, y al tratarse de estimaciones horarias de radiación solar, la calidad de las estimaciones obtenidas con modelos lineales puede verse reducida. Para comprobar que las redes neuronales son más adecuadas en estos casos, en este trabajo probamos regresión lineal para comparar los resultados con los obtenidos con redes neuronales.

$\mathrm{Al}$ incluir las variables con información del pasado (lagged variables) en el vector de entrada, existe una correlación lineal fuerte entre las variables de entrada. Por este motivo, los sistemas lineales involucrados pueden ser mal condicionados, es decir que producen una fuerte variación en la salida ante pequeños cambios en la entrada, haciendo que la solución no sea adecuada. Para evitar este problema utilizamos pseudoinversa de Moore-Penrose (Eldén, 2007), que es capaz de obtener buenas soluciones incluso en presencia de sistemas mal condicionados. Las variables de entrada utilizadas son las mismas tanto en el caso de redes neuronales y el de regresión lineal, pero en el segundo caso la normalización de los datos no es necesaria.

Con el objetivo de evaluar estadísticamente el desempeño de los modelos implementados, se analiza el error a través de distintas métricas utilizadas en la literatura, comparando los valores de radiación solar calculada $\left(Y_{i}\right)$ con la radiación solar medida $\left(T_{i}\right)$. Las métricas de error utilizadas son Root Mean Squared Error o RMSE, que al estar expresado en la misma unidad de medida que la variable a estimar facilita su interpretación; Root Mean Squared Error Porcentual o RMSE\%, expresado como porcentaje; Mean Bias Error o $M B E$, que permite saber si existe una subestimación o una sobreestimación, analizando su signo; Coeficiente de Correlación Lineal de Pearson $R$, que ayuda a determinar el grado con que los datos siguen la tendencia general del modelo.

\section{Resultados y Discusión}

Los niveles de error obtenidos utilizando únicamente el modelo empírico, como así también aquellos obtenidos combinando el modelo empírico con regresión lineal y con redes neuronales se muestra en la Tabla 2. Como podemos observar, la combinación del modelo empírico y redes neuronales fue la que tuvo un mejor desempeño, logrando niveles de error mucho más bajo que los otros métodos. Esto se ve reflejado en todas las métricas de error en todas las estaciones meteorológicas, logrando en promedio un error RMSE\% de 8.3, lo que implica una reducción de 20.9\% de error respecto al modelo empírico únicamente. En segundo lugar se encuentra la 
combinación de modelo empírico con regresión lineal que, si bien no logra el mejor resultado, permite una reducción del $18.194 \%$ respecto al modelo empírico solo. Por otro lado, si comparamos los niveles de error en el conjunto de entrenamiento y validación podemos ver que no hay una diferencia significativa, lo que permite afirmar que el modelo generado es estable y no requiere ajustes adicionales.

En la Figura 3 se muestran los diagramas de dispersión correspondiente a los datos experimentales (valores reales de radiación solar) y las estimaciones realizadas con la red neuronal, para el conjunto de entrenamiento y validación respectivamente. Se muestran sólo los datos de El Colmenar, ya que para las otras estaciones meteorológicas los resultados son similares. Se puede observar que existe una leve sub-estimación para valores de radiación entre 800 y 1000 [W/m2] que se incrementa gradualmente a partir de los $1000[\mathrm{~W} / \mathrm{m} 2]$. Esto se ve reflejado en el error MBE en la Tabla 2, lo que da indicios de que el método presenta una dificultad para estimar los picos de radiación solar, presentes sobre todo en época de verano.
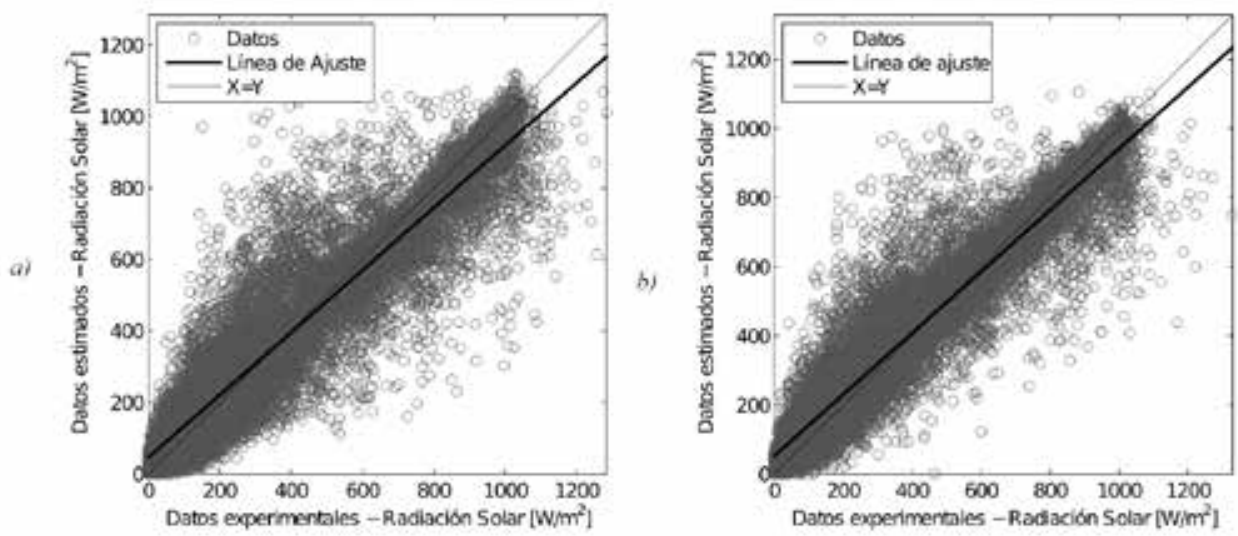

Figura 3: Resultados obtenidos combinando modelo empírico y redes neuronales para El Colmenar a) Datos de entrenamiento, b) Datos de validación.

Por último, en la Figura 4 se muestra una comparativa de los perfiles de curva de radiación solar real tomada en la estación meteorológica de El Colmenar, superpuesta a las curvas generadas con los valores estimados. Podemos ver que ambas curvas son muy similares salvo por algunos picos, por lo que la tendencia general de los datos es correctamente reproducida por el modelo propuesto. 


\section{Conclusiones}

En este trabajo se hicieron estimaciones de radiación solar combinando un modelo empírico con redes neuronales artificiales. Las variables utilizadas incluyen variables climáticas de fácil acceso como la temperatura ambiente, humedad relativa y presión atmosférica, y parámetros asociados al sitio de la estimación como la latitud y longitud. Pudimos ver que la metodología utilizada además de dar buenos resultados para estimar radiación solar diaria, resultó también ser eficaz para estimar la radiación solar a nivel horario. A pesar de que las variables utilizadas son limitadas en cantidad, se obtuvo una precisión adecuada para las estimaciones. Pudimos comprobar que el modelo empírico genera estimaciones con altos niveles de error (con un RMSE del orden del 29.2\%), pero al utilizar estas estimaciones con una red neuronal se reduce significativamente el error a $7.8 \%$. Por otro lado, también se pudo verificar que las redes neuronales, a pesar de que tienen un costo computacional más elevado, pudieron captar mejor tendencia general de los datos que la regresión lineal.

La metodología utilizada además de brindar buenos resultados, presenta otras ventajas. Por un lado es posible utilizarla para predecir los valores de radiación solar en un horizonte a corto y mediano plazo. Esto es posible debido a que es muy sencillo contar con pronósticos extendidos del clima, donde las predicciones de las variables climáticas utilizadas en este trabajo son muy comunes. Por lo tanto es factible aplicar esta metodología con datos de pronósticos permitiendo obtener predicciones de la radiación solar. Además, el procedimiento general descrito en este trabajo permite la adición de otras variables, o cambiar el modelo empírico por otro más preciso, para mejorar la calidad de los resultados. Sin embargo, esto dependerá de la disponibilidad de estas variables en el sitio donde se quiera aplicarla. 


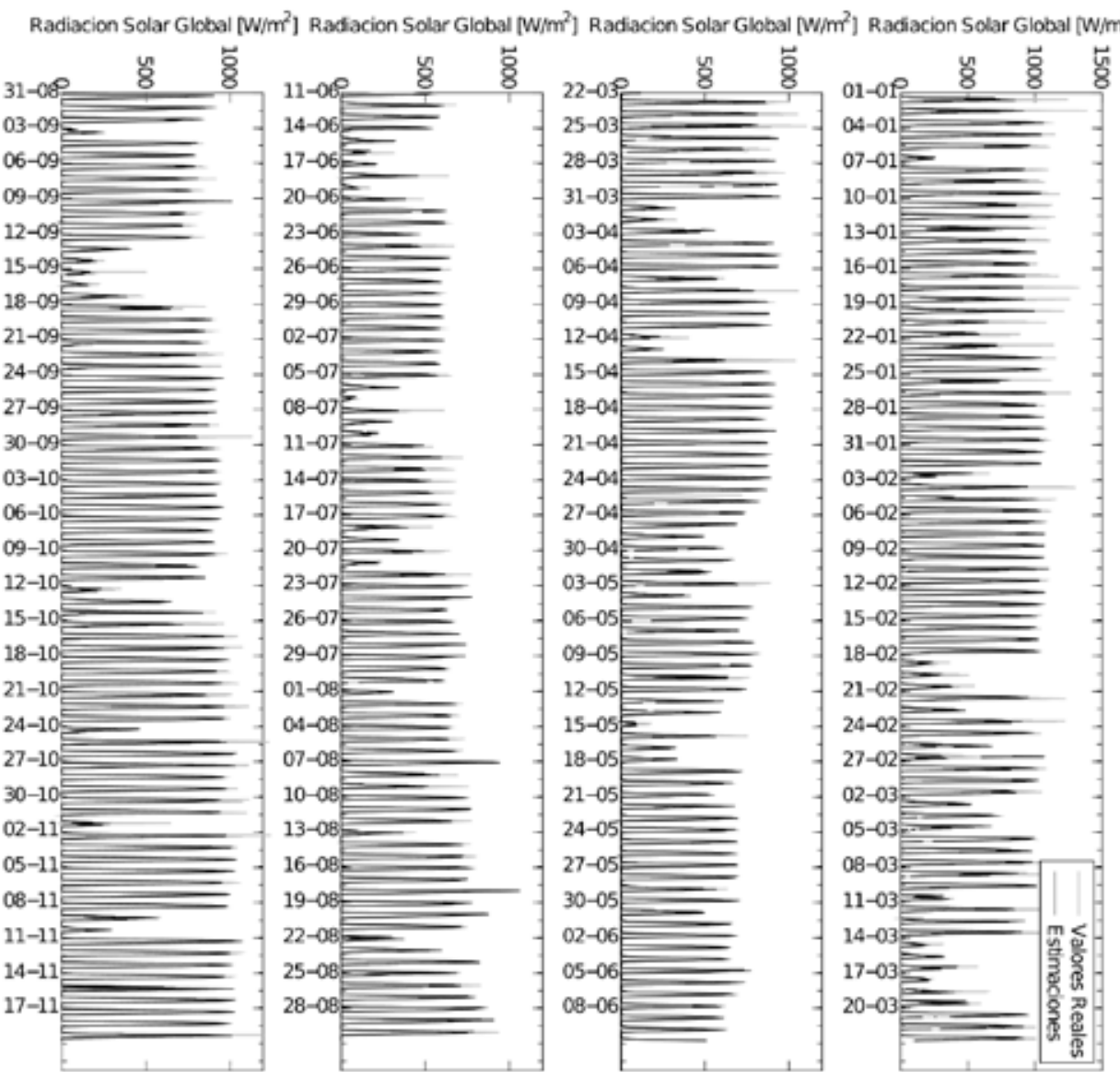

Figura 4: Perfiles de curva de mediciones y estimaciones de Radiación solar en El Colmenar, para datos de validación (año 2013).

\section{Agradecimientos}

Este trabajo fue soportado parcialmente por subsidios UTI4015 y UTN3870. También deseamos extender los agradecimientos a la Estación Experimental Obispo Colombres (E.E.A.O.C.) por proporcionar los datos necesarios para la reali0zación del trabajo. 


\section{Referencias}

Ahmad, M. J., \& Tiwari, G. (2011). Solar radiation models-A review. International Journal of Energy Research, 35(4), 271-290.

Cao, J., \& Lin, X. (2008). Study of hourly and daily solar irradiation forecast using diagonal recurrent wavelet neural networks. Energy Conversion and Management, 49(6), 1396-1406.

Eldén, L. (2007). Matrix Methods in Data Mining and Pattern Recognition. Philadelphia, PA, USA: Society for Industrial and Applied Mathematics.

Elminir, H. K., Azzam, Y. A., \& Younes, F. I. (2007). Prediction of hourly and daily diffuse fraction using neural network, as compared to linear regression models. Energy, 32(8), 1513-1523.

Furlan, C., De Oliveira, A. P., Soares, J., Codato, G., \& Escobedo, J. F. (2012). The role of clouds in improving the regression model for hourly values of diffuse solar radiation. Applied Energy, 92, 240-254.

Gilani, S. I.-H., Dimas, F. A. R., Shiraz, M., \& others. (2011). Hourly solar radiation estimation using Ambient Temperature and Relative Humidity data. International Journal of Environmental Science and Development, 2(3), 188193.

Gorgan, B., Notingher, P., Wetzer, J., Verhaart, H., Wouters, P., \& Van Schijndel, A. (2012). Influence of solar irradiation on power transformer thermal balance. IEEE Transactions on Dielectrics and Electrical Insulation, 19(6), 1843-1850.

Hagan, M. T., \& Menhaj, M. B. (1994). Training feedforward networks with the Marquardt algorithm. Neural Networks, IEEE Transactions on, 5(6), 989-993.

Hernández-Travieso, J. G., Travieso, C. M., Alonso, J. B., \& Dutta, M. K. (2014). Solar radiation modelling for the estimation of the solar energy generation. En Contemporary Computing (IC3), 2014 Seventh International Conference on (pp. 536-541).

Ji, W., Chan, C., Loh, J., \& Chen, L. (2009). Solar Radiation Prediction Using Statistical Approaches. En Information, Communications and Signal Processing, ICICS 2009 (pp. 1-5). IEEE.

Jimenez, V. A., Barrionuevo, A., Will, A., \& Rodriguez, S. (2016). Neural Network for Estimating Daily Global Solar Radiation Using Temperature, Humidity and Pressure as Unique Climatic Input Variables. Smart Grid and Renewable Energy, (7), 94-103. http://doi.org/http://dx.doi.org/10.4236/sgre.2016.73006 
Jimenez, V. A., Will, A., Rodriguez, S. A., \& Lamelas, C. (2014). Imputación de Datos Climáticos Utilizando Algoritmos Genéticos Niching. En Acta de la XXXVII Reunión de Trabajo de la Asociación Argentina de Energías Renovables y Medio Ambiente (Vol. 2, pp. 11139-11148).

Khatib, T., Mohamed, A., Sopian, K., \& Mahmoud, M. (2012). Assessment of artificial neural networks for hourly solar radiation prediction. International journal of Photoenergy, 2012.

Mellit, A., Eleuch, H., Benghanem, M., Elaoun, C., \& Pavan, A. M. (2010). An adaptive model for predicting of global, direct and diffuse hourly solar irradiance. Energy Conversion and Management, 51(4), 771-782.

Noorian, A. M., Moradi, I., \& Kamali, G. A. (2008). Evaluation of 12 models to estimate hourly diffuse irradiation on inclined surfaces. Renewable energy, 33(6), 1406-1412.

Pandey, P. K., \& Soupir, M. L. (2012). A new method to estimate average hourly global solar radiation on the horizontal surface. Atmospheric Research, 114115(0), 83-90.

Reikard, G. (2009). Predicting solar radiation at high resolutions: A comparison of time series forecasts. Solar Energy, 83(3), 342-349.

Sayago, S., Bocco, M., Ovando, G., \& Willington, E. (2011). Radiación solar horaria: modelos de estimación a partir de variables meteorológicas básicas. Avances en Energías Renovables y Medio Ambiente, 15, 51-57.

Sfetsos, A., \& Coonick, A. (2000). Univariate and multivariate forecasting of hourly solar radiation with artificial intelligence techniques. Solar Energy, 68(2), 169-178.

Sharma, S. K., \& Chandra, P. (2010). Constructive neural networks: a review. International journal of engineering science and technology, 2(12), 7847-7855.

Spokas, K., \& Forcella,Frank. (2006). Estimating hourly incoming solar radiation from limited meteorological data. Weed Science, 54, 182-189.

Teke, A., Yıldırım, H. B., \& Çelik, Ö. (2015). Evaluation and performance comparison of different models for the estimation of solar radiation. Renewable and Sustainable Energy Reviews, 50, 1097-1107.

Will, A., Bustos, J., Bocco, M., Gotay, J., \& Lamelas, C. (2013). On the use of niching genetic algorithms for variable selection in solar radiation estimation. Renewable Energy, 50, 168-176. 
Yadav, A. K., \& Chandel, S. (2014). Solar radiation prediction using Artificial Neural Network techniques: A review. Renewable and Sustainable Energy Reviews, 33, 772-781.

Yu, H., \& Wilamowski, B. M. (2011). Levenberg-marquardt training. Industrial Electronics Handbook, 5, 12-1. 
